\title{
Towards Atomic-Scale Fabrication in Silicon
}

Bethany M. Hudak ${ }^{1,2}$, Stephen Jesse ${ }^{2,3}$, Jiaming Song ${ }^{1}$, Albina Borisevich ${ }^{1}$, Paul C. Snijders ${ }^{1}$, Sergei V. Kalinin $^{2,3}$, and Andrew R. Lupini ${ }^{1,2}$

1. Materials Science and Technology Division, Oak Ridge National Laboratory, Oak Ridge, TN, USA

2. The Institute for Functional Imaging of Materials, Oak Ridge National Laboratory, Oak Ridge, TN, USA

3. The Center for Nanophase Materials Sciences, Oak Ridge National Laboratory, Oak Ridge, TN, USA

The advance of modern technology requires the ability to produce ever-shrinking devices, in some cases reaching atomic scales or relying on quantum effects. This demand for quantum devices requires fabrication on the atomic scale to guarantee precise control of crystalline layers, active dopant placement, and to avoid or understand atomic-scale defects. However, current techniques for nanoscale fabrication in the semiconductor industry lack the capability to fabricate on the atomic scale. The current state of the art in atomic fabrication includes the positioning of single atoms [1] and surface lithography [2] using scanning tunnelling microscopy (STM) and atomic force microscopy (AFM) on twodimensional surfaces $[3,4]$, but an active quantum device would require sub-surface dopant positioning [5]. For the development of quantum devices, these techniques are limited in two ways: 1) There is still a need for atomic-scale fabrication of ancillary structures, and 2) The atomic manipulation is limited to surfaces. For quantum fabrication, the goal is to construct semiconductor devices with atomic-layer precision and to control the placement of active subsurface dopants. Scanning transmission electron microscopy (STEM) has recently been demonstrated as a technique for the epitaxial growth of material at a crystalline-amorphous (CA) interface [6,7]. Here we show the ability to crystallize, amorphize, remove material, and direct dopants, which form important steps toward quantum device fabrication.

Through the use of the atomically-precise probe of a STEM, we can controllably crystallize (Fig. 1a-b), amorphize (Fig. 1c-d), and drill holes (Fig. 1e-f) in silicon at the atomic scale. As in conventional STEM imaging, the electron beam is focused to an atomically sharp probe and rastered across the sample. As the beam scans into the amorphous region, there is a clear formation of crystalline Si in epitaxial registry with the Si substrate. By increasing the beam current, we can transform the crystalline Si substrate to amorphous $\mathrm{Si}$ or drill holes to remove $\mathrm{Si}$. To further control the crystallization process, we have developed a feedback and control system to guide atomic layer by atomic layer transformation. By scanning the beam parallel to the CA interface and calculating the amplitude of the Fourier transform of the STEM signal, we determine the degree of crystallinity. The beam is then automatically advanced one atomic layer at a time when the desired transformation is achieved. Because most semiconducting devices rely on doped $\mathrm{Si}$, it is then essential to determine how dopants are affected by the manipulation process. Using a sample with a high concentration of Bi-dopants, we can directly image how this technique affects sub-surface dopants. By scanning the beam, similarly to the crystallization method, we can move dopants perpendicular to the fast-scan direction of the beam, along multiple Si crystal axes.

The major challenges for the fabrication of quantum devices are the ability to control semiconductor growth on the nanoscale and to accurately position subsurface dopants. Here, we demonstrate the atomic manipulation of $\mathrm{Si}$ - the most important industrial semiconductor - with the ability to crystallize, amorphize, and remove material on the atomic scale, as well as position functional dopants. With the real-time monitoring afforded by STEM, device architectures can be grown and edited in situ at the atomic scale, providing an important step toward fabrication of atomic-scale devices. [8] 
References:

[1] D. M. Eigler, E. K. Schweizer, Nature 344 (1990), p. 524-526.

[2] M. Fuechsle et al., Nat. Nanotechnol. 7 (2012), p. 242-246

[3] O. Custance, R. Perez, S. Morita, Nat. Nanotechnol. 4 (2009), p. 803-810.

[4] N. Oyabu, Ó. Custance, I. Yi, Y. Sugawara, S. Morita, Phys. Rev. Lett. 90 (2003), p. 176102.

[5] B. E. Kane, Nature. 393, (1998), p. 133-137.

[6] S. Jesse et al., Small. 11 (2015), p. 5895-5900.

[7] N. Jiang et al., MRS Bull. 42, (2017), p. 653-659.

[8] This research was partially (B.M.H., J.S., P.C.S) supported by the Laboratory Directed Research and Development program of the Oak Ridge National Laboratory, managed by UT-Battelle, LLC, for the U.S. Department of Energy. Part of this work (S.J., S.V.K.) was supported by the Center for Nanophase Materials Sciences, which is a US DOE Office of Science User Facility. A.B. and A.R.L. were supported by the U.S. Department of Energy, Office of Science, Basic Energy Sciences, Division of Materials Science and Engineering.

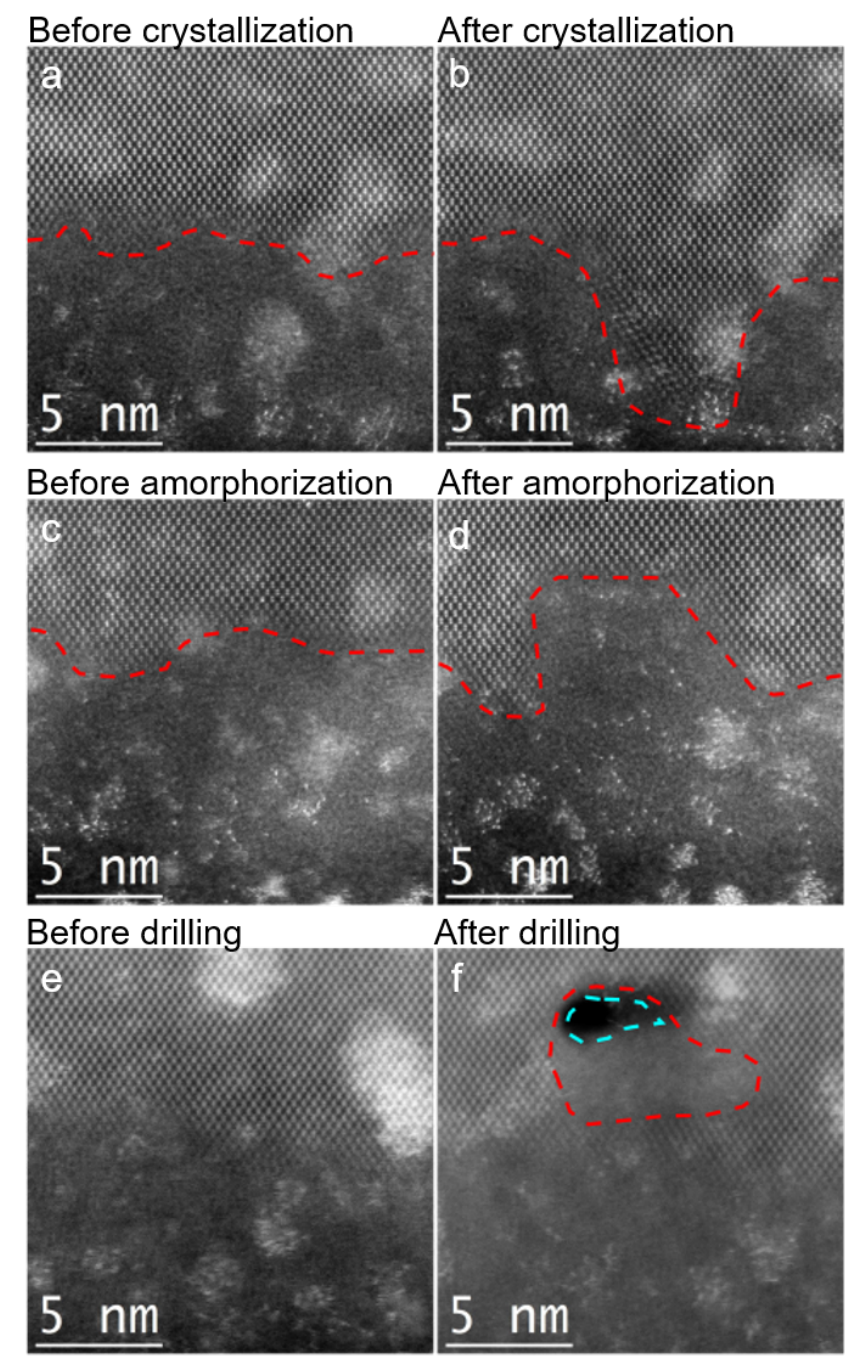

Figure 1. The beam of a STEM is used as a tool for silicon fabrication. (a-b) By scanning from the crystal substrate into the amorphous region, epitaxial Si is grown. (c-d) Under increased beam current conditions and scanning from the amorphous region into the crystalline region, the crystal becomes amorphous. (e-f) When the beam current is increased further, the beam is used to remove material and drill holes into the crystalline substrate. The red dashed lines indicate the CA interface, and the blue dashed line highlights the hole drilled through the $\mathrm{Si}$ crystal. 